1996

\title{
Classical Singularities In Chaotic Atom-Surface Scattering
}

\author{
S. Miret-Artés
}

J. Margalef-Roig

R. Guantes

F. Borondo

Charles Jaffé

Follow this and additional works at: https://researchrepository.wvu.edu/faculty_publications

\section{Digital Commons Citation}

Miret-Artés, S.; Margalef-Roig, J.; Guantes, R.; Borondo, F.; and Jaffé, Charles, "Classical Singularities In Chaotic Atom-Surface Scattering" (1996). Faculty Scholarship. 485.

https://researchrepository.wvu.edu/faculty_publications/485 


\title{
Classical singularities in chaotic atom-surface scattering
}

\author{
S. Miret-Artés and J. Margalef-Roig \\ Instituto de Matemáticas y Física Fundamental, Consejo Superior de Investigaciones Científicas, Serrano 123, 28006 Madrid, Spain \\ R. Guantes and F. Borondo \\ Departamento de Química, C-IX, Universidad Autónoma de Madrid, Cantoblanco-28049 Madrid, Spain \\ Charles Jaffé \\ Deparment of Chemistry, West Virginia University, Morgantown, West Virginia 26506
}

(Received 4 June 1996)

\begin{abstract}
In this paper we show that the diffraction condition for the scattering of atoms from surfaces leads to the appearance of a distinct type of classical singularity. Moreover, it is also shown that the onset of classical trapping or classical chaos is closely related to the bifurcation set of the diffraction-order function around the surface points presenting the rainbow effect. As an illustration of this dynamic, application to the scattering of $\mathrm{He}$ atoms by the stepped $\mathrm{Cu}(115)$ surface is presented using both a hard corrugated one-dimensional wall and a soft corrugated Morse potential. [S0163-1829(96)05639-1]
\end{abstract}

Surface rainbows correspond to extrema, either maxima or minima, of the classical deflection (CD) function, which is defined as the variation of the final or outgoing angle of the scattering particles with respect to the impact points on the surface (expressed in terms of the impact parameter). Within the classical framework, and under certain conditions, these extrema lead to singularities in diffraction patterns usually known as caustics. In quantum mechanics, caustics are replaced by finite scattering intensities in all Bragg directions. Garibaldi et al. ${ }^{1}$ and Berry ${ }^{2}$ showed that classical rainbow patterns provide the envelope of the quantum-mechanical diffraction peak intensities. Care must be exercised in the assignment of experimentally observed features as rainbows. ${ }^{3}$ This is due to the fact that, in addition to the rainbow singularities, other classical and quantummechanical effects occur that lead to features that are easily mistaken for rainbows. These effects often take place when the classical dynamics associated with the scattering process is chaotic. In these circumstances, the scattering particle is trapped on the surface for a finite time. This temporary (vibrational) trapping has been conjectured to be associated with selective adsorption resonances. However, as has been recently stated, ${ }^{4}$ the relation between trapping and these resonances needs further investigation to clearly understand both phenomena. In addition to the occurrence of such quantummechanical resonances, we show in this work that trapping is related to a type of classical singularity. This appears in the transformation from the CD function to the diffraction order (DO) function, which was originally introduced by Miller et $a .^{5}$ in his theory of the classical S-matrix for elastic atomsurface scattering. This function gives the parallel momentum transfer in the scattering process for each value of the impact parameter.

For any incident energy, the surface rainbow angles are dependent on the incident scattering angle and occur at certain impact parameters, which correspond to the inflection points of the surface. As the incident scattering angle is increased, these angles also increase. The onset of classical trapping, that is, classical chaos, takes place when a surface rainbow angle reaches $\pm \pi / 2$, according to Ref. 4 . A rainbow angle of $\pm \pi / 2$ implies that the scattering particle is traveling parallel to the surface and likely will encounter the surface more than once. As will be shown later, if we analyze plots of the incident angle versus the impact parameter, above a given threshold of the incident angle, not only do the inflection points of the surface lead to this classical trapping, but, also, more impact parameters contribute to it. In these plots, two well-separated regions delimited by the two branches of a parabola are observed in such a way that the inner region corresponds to multiple scattering events and the outer region to single scattering events. The points of this parabola define the singularity that we are discussing in this paper.

In order to demonstrate the origin of this singularity, we consider the classical in-plane scattering intensity as formulated in Ref. 5:

$$
I_{J}\left(E_{i}, \theta_{i}\right)=\sum_{j}\left|\frac{d J\left(b_{j} ; E_{i}, \theta_{i}\right)}{d b}\right|^{-1}
$$

where $E_{i}$ is the incident energy, $\theta_{i}$ is the incident scattering angle, $b$ is the normalized impact parameter (defined by $b=x / a$ ), and $a$ is the surface unit-cell length considered one-dimensional along the $x$ direction; the sum is over all impact parameters $b_{j}$ that contribute to a given integer value, $J$, of the DO function, $J\left(b ; E_{i}, \theta_{i}\right)$. This function arises as an alternative way to express the diffraction condition and can be written as

$$
J\left(b ; E_{i}, \theta_{i}\right)=a \frac{\sqrt{2 m E_{i}}}{2 \pi \hbar}\left[\sin \theta_{f}(b)-\sin \theta_{i}\right],
$$

$\theta_{f}(b)$ being the $\mathrm{CD}$ function. Equation (2) is thus more adapted to a classical analysis and along with Eq. (1) gives the envelope of the diffraction pattern. In order to obtain the intensity in the different Bragg directions, one has to evaluate this function for integer values of the diffraction order, 
$J$. The scattering intensity will have singularities whenever the derivative of the DO function with respect to $b$ is zero or, using the chain rule, when

$$
\left(\frac{d J\left(\theta_{f} ; E_{i}, \theta_{i}\right)}{d \theta_{f}}\right)\left(\frac{d \theta_{f}(b)}{d b}\right)=0 .
$$

Clearly, if either of these two factors or both are equal to zero, they give rise to different kinds of singularities in the scattering intensity. Zeros of the first factor are responsible for the new singularity that we are describing in this paper, and the zeros of the second factor correspond to the wellknown surface rainbows. We consider these two factors separately.

The second factor in Eq. (3) is the derivative of the CD function with respect to $b$. If we want to perform this derivative analytically, it is necessary to specify a simple interaction model for this scattering. For this purpose, we describe the surface by a hard corrugated one-dimensional wall. The diffraction amplitudes in the eikonal approximation are expressed as

$$
A_{g}=\frac{1}{a} \int d x \exp \left\{-i\left[g x+q_{g z} \xi(x)\right]\right\},
$$

where $g=(2 \pi n / a, 0)$ is the reciprocal lattice vector with $n$ an integer, $q_{g z}$ is the momentum transfer of the particles in the $z$ direction, and $\xi(x)$ is the corrugation function. In the framework of this approximation, multiple scattering is neglected. The $\mathrm{CD}$ function is then obtained by evaluating the integral of Eq. (4) in the stationary phase approximation together with the diffraction condition, Eq. (2), to be

$$
\theta_{f}(b)=\theta_{i} \pm 2\left|\tan ^{-1}\left[\xi^{\prime}(b) / a\right]\right|,
$$

where the + sign is used when the $\tan ^{-1}$ function gives negative angles and the - sign otherwise. Thus, the second factor in Eq. (3) is

$$
\left(\frac{d \theta_{f}(b)}{d b}\right)=\frac{2\left|\xi^{\prime \prime}(b) / a\right|}{1+\left[\xi^{\prime}(b) / a\right]^{2}} .
$$

From this equation, it is seen that a surface rainbow occurs for each inflection point of the surface corrugation function, $\xi^{\prime \prime}\left(b^{*}\right)=0$, where $b^{*}$ designates the inflection points. As it is well known, this singularity is related to the topology of the surface.

The first factor in Eq. (3) is easily obtained by differentiating Eq. (2) and this yields

$$
\left(\frac{d J\left(\theta_{f} ; E_{i}, \theta_{i}\right)}{d \theta_{f}}\right)=a \frac{\sqrt{2 m E_{i}}}{2 \pi \hbar} \cos \theta_{f} .
$$

The singularity occurs when the deflection angle is again equal to $\pm \pi / 2$ (onset of classical trapping) but for impact parameters, in general, different from $b^{*}$. Prior to the onset of classical trapping this singularity does not occur. It arises as a consequence of the diffraction condition and a classical image of this singular behavior could be given by the skipping stones on a river. Thus in order to skip a stone on a river it is necessary not only to get the incident angle correct but also to reach certain impact points on the water surface.
TABLE I. Potential energy surface for the scattering of $\mathrm{He}$ from the $\mathrm{Cu}(115)$ surface at $63 \mathrm{meV}$ (Ref. 6).

Potential energy: $V(x, z)=V_{M}(z)+V_{C}(x, z)$

Morse potential: $V_{M}(z)=D\left(1-e^{-\alpha z}\right)^{2}$

$D=6.35 \mathrm{meV}, \alpha=1.05 \AA^{-1}$

Coupling potential: $V_{C}(x, z)=V_{z}(z) V_{x}(x)$

$V_{z}(z)=D \exp (-2 \alpha z)$

$V_{x}(x)=\sum_{n=1}^{2}\left[r_{n} \cos \frac{2 n \pi x}{a}+s_{n} \sin \frac{2 n \pi x}{a}\right]$

Fourier coefficients at $63 \mathrm{meV}$ :

$r_{1}=0.2147, s_{1}=-0.0252$,

$r_{2}=0.0131, s_{2}=-0.0044$

Unit cell length for $\mathrm{Cu}(115): a=6.625 \AA$

The singularity condition can be also expressed in a very useful way in terms of $\theta_{i}$ and $b$ by substituting Eq. (5) in Eq. (7) and equating the result to zero. Then the locus of this singularity is given by the following planar curve

$$
\tan \theta_{i}(b)= \pm \frac{1-\left[\xi^{\prime}(b) / a\right]^{2}}{2\left|\xi^{\prime}(b) / a\right|} .
$$

Notice that this function has a minimum at $b=b^{*}$ with $\theta_{i}=\theta_{i}^{*}$ Moreover, at this point, in which both factors of Eq. (3) vanish simultaneously, the second derivative of the DO function with respect to $b$ is also equal to zero. Therefore the set of conditions (i) $\xi^{\prime \prime}\left(b^{*}\right)=0$ but $\xi^{\prime \prime \prime \prime}\left(b^{*}\right) \neq 0$, and (ii) Eq. (8) with $b=b^{*}$, define the bifurcation set of the DO function $\left[J^{\prime}\left(b^{*}\right)=J^{\prime \prime}\left(b^{*}\right)=0\right]$. This point also coincides with the onset of classical trapping and chaos. ${ }^{4}$ For incident angles above the critical value $\theta_{i}^{*}$ the rainbow singularity bifurcates into two branches, the new singularities, for which only Eq. (8), and not condition (i), is fulfilled.

In order to clearly illustrate this dynamics, we consider a detailed analysis of the scattering of $\mathrm{He}$ atoms from the stepped $\mathrm{Cu}(115)$ surface for an incident energy of $63 \mathrm{meV}$. Rainbow diffraction patterns have been observed experimentally for this surface, ${ }^{6,7}$ and theoretical calculations have been also reported within the close-coupling ${ }^{3,8}$ and semiclassical $S$-matrix ${ }^{3,9}$ formalisms. The interaction potential model for this surface and at this energy has been taken from the literature ${ }^{6}$ and corresponds to a fitting to experimental results. The corresponding parameters are given in Table I.

In Fig. 1 the bifurcation diagram [Eq. (8)] corresponding to the one-dimensional corrugation function, $\xi(x)=V_{x}(x)$, given in Table I is shown. Here $b^{*}=0.22$ and the onset of classical trapping is predicted at $\theta_{i}^{*}=68.73^{\circ}$. The region above the curve corresponds to conditions of multiple scattering events or trapping, and the region below to single scattering events. Also, in the inset of this figure we show the classical diffraction pattern corresponding to the minimum of the curve. As can be seen the asymptotic behavior is very different for both singularities. In the rainbow singularity (left) the tendency is controlled by surface structural properties [see Eq. (6)], while in the new singularity (right) the asymptotic behavior is of cosine type [see Eq. (7)].

In Fig. 2 we show the DO functions obtained from Eq. (2) (dashed line) and by numerical integration of the Hamilton equations (solid line) at $\theta_{i}=28^{\circ}$. The differences in both 


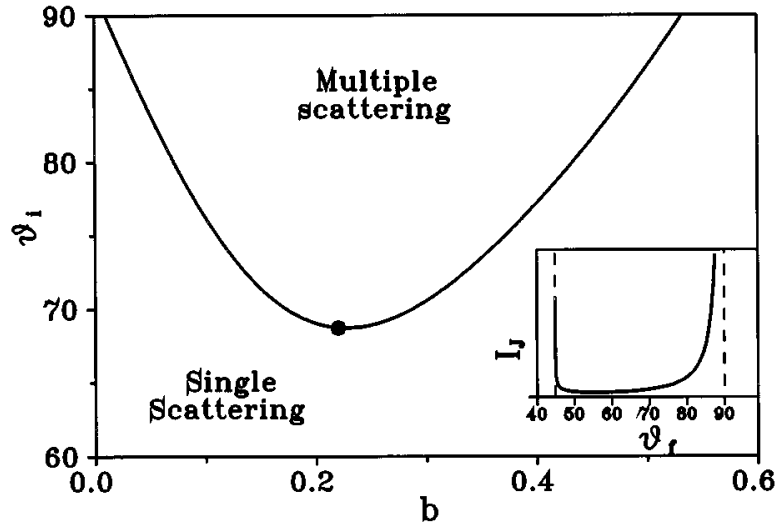

FIG. 1. Bifurcation diagram for the singularities in the He$\mathrm{Cu}(115)$ scattering. The location of the rainbow singularity, $b^{*}=0.22, \theta_{i}^{*}=68.73^{\circ}$, is marked with a full circle. Above this point the rainbow singularity bifurcates, giving rise to the two singularity branches described in the text. In the inset the classical diffraction pattern corresponding to the bifurcation point is shown.

curves are only due to the presence of the attractive part of the potential. Although the numerical agreement is not good, the main qualitative features of the dynamics are rather well reproduced in the analytical solution given by the eikonal approximation. At the same incident energy, we display in Fig. 3(a) the analytical CD functions [Eq. (5)] and in Fig. 3(b) the analytical DO functions [Eq. (2)] at three different incident angles: $62^{\circ}$ (dotted line), $68.73^{\circ}$ (solid line) and $75^{\circ}$ (dashed line), respectively. Whereas the $\mathrm{CD}$ functions do not present any qualitative change (number of extrema), the DO functions present around the maximum drastic changes. From a large plateau we pass to the occurrence of three extrema. The minimum value of $J$ corresponds to the old maximum at $b^{*}$. Numerically the onset of chaos appears at $54^{\circ}$ and not at $68.73^{\circ}$; this difference is again due to the presence of the soft interaction potential.

To our knowledge, the effect described in this paper has not been observed experimentally in any diffraction experi-

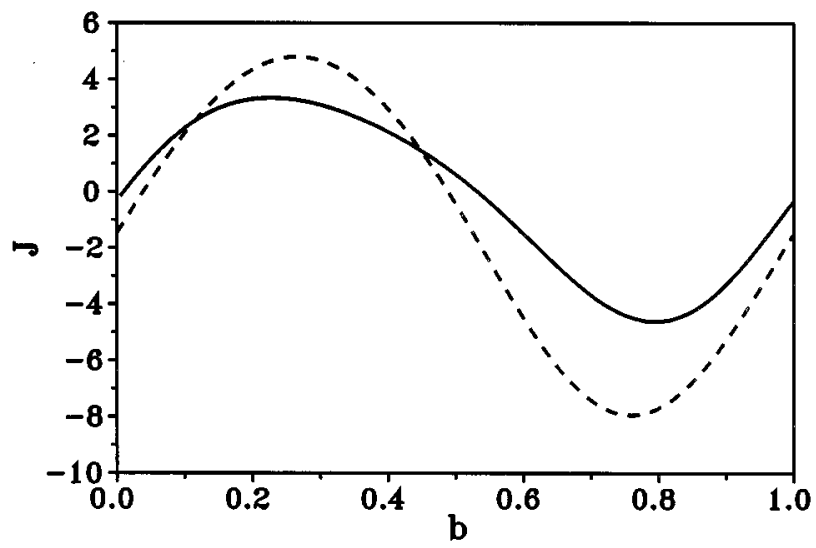

FIG. 2. Analytical (solid line) and numerical (dashed line) DO functions for the scattering of $\mathrm{He}$ from $\mathrm{Cu}(115)$ at an incident energy of $63 \mathrm{meV}$ and incident angle of $28^{\circ}$.

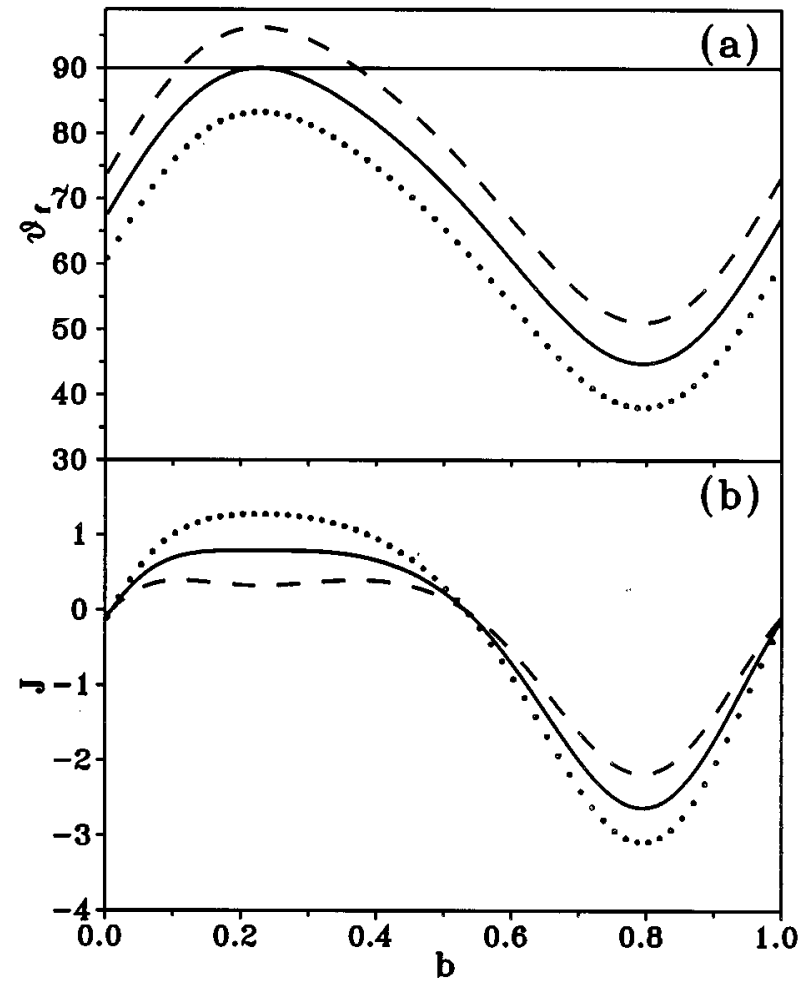

FIG. 3. Analytical CD function (a) and DO function (b) at three incident angles: $62^{\circ}$ (dotted line), $68.73^{\circ}$ (solid line) and $75^{\circ}$ (dashed line). The incident energy for the scattering of He from $\mathrm{Cu}(115)$ is $63 \mathrm{meV}$.

ment. To a large degree this could be due to technical difficulties but they are easily surmounted. In order to observe it, one needs to place the detector parallel to the surface and then to vary the incident scattering angle in order to find its critical value $\theta_{i}^{*}$; the outgoing beam being a Bragg peak. This would require us to change the typical experimental setup, where the angle between the source and the detector $\left(\theta_{\mathrm{SD}}\right)$ is usually around $\pi / 2$, to a more planar configuration (in general greater than $100^{\circ}$ ). ${ }^{10}$ For example, in our case where the critical incident angle value is $54^{\circ}$, with an incident wave vector of $5 \AA^{-1}$ the (10) diffraction peak should present such an effect by using an experimental configuration with $\theta_{\mathrm{SD}}=144^{\circ}$. Similar features to those discussed for the rainbow effect are expected here in the diffraction intensities. Finally, due to the fact that this singularity has its origin in the diffraction condition we think that it could also be observed in any scattering of low energy particles (such as neutrons, electrons, etc.) from surfaces.

\section{ACKNOWLEDGMENTS}

This work has been supported in part by DGICYT (Spain) under Grants Nos. PB89-122, PB92-53, and PB92-181, and by the NSF (USA) under Grant No. RII-8922106. R.G. gratefully acknowledges a grant from the Ministerio de Educación y Ciencia (Spain). 
${ }^{1}$ U. Garibaldi, A. G. Levi, R. Spadacini, and G. E. Tommei, Surf. Sci. 48, 649 (1975).

${ }^{2}$ M. V. Berry, J. Phys. A 8, 566 (1975).

${ }^{3}$ R. Guantes, F. Borondo, C. Jaffé, and S. Miret-Artés, Surf. Sci. 338, L863 (1995).

${ }^{4}$ F. Borondo, C. Jaffé, and S. Miret-Artés, Surf. Sci. 317, 211 (1994).

${ }^{5}$ R. I. Massel, R. P. Merril, and W. H. Miller, J. Chem. Phys. 64, 45 (1976).
${ }^{6}$ G. Gorse, B. Salanon, F. Fabre, A. Kara, J. Perreau, G. Armand, and J. Lapujoulade, Surf. Sci. 147, 611 (1984).

${ }^{7}$ S. Miret-Artés, J. P. Toennies, and G. Witte, Phys. Rev. B (to be published).

${ }^{8}$ M. Hernandez, S. Miret-Artés, P. Villarreal, and G. DelgadoBarrio, Surf. Sci. 290, L693 (1993).

${ }^{9}$ R. Guantes, F. Borondo, C. Jaffé, and S. Miret-Artés, Phys. Rev. B 53, 14117 (1996).

${ }^{10} \mathrm{~J}$. P. Toennies (private communication). 